Radiologe 2019 · 59:885-893

https://doi.org/10.1007/s00117-019-0567-9

Online publiziert: 4. Juli 2019

(c) Der/die Autor(en) 2019
D. Beitzke

Universitätsklinik für Radiologie und Nuklearmedizin, Medizinische Universität Wien, Wien, Österreich

\title{
Sichere MRT-Untersuchung bei Patienten mit Herzschrittmacher und ICD
}

Lange Zeit galten MRT-Untersuchungen von Patienten mit Schrittmachern (SM) bzw. implantierbaren Kardioverter-Defibrillatoren (ICD) als kontraindiziert. Als zu groß und zu unberechenbar wurde die Gefahr der Interaktion zwischen diesen elektrisch aktiven Geräten und den unterschiedlichen Feldern eines MRGeräts angesehen.

Innerhalb der letzten zwei Jahrzehnte wurde jedoch reichlich Evidenz geschaffen, welche zeigte, dass sichere MRT-Untersuchungen unter bestimmten Voraussetzungen bei diesem Patientengut möglich sind. Zusätzlich gab es Weiterentwicklungen im Schrittmacherdesign betreffend Materialen, Sensoren und Software, welche dazu führten, dass viele der heute am Markt befindlichen Schrittmacher als "MRconditional“ (MRc), also „bedingt MR-sicher" eingestuft werden können. Der folgende Artikel bietet eine Übersicht über die vorhandene Literatur und soll einen praxisnahen Leitfaden für die sichere MRTUntersuchung von Schrittmacherpatienten bieten.

\section{Warum ist eine MRT bei Schrittmacherpatienten notwendig?}

Die MRT als nichtinvasive Untersuchungsmodalität ist frei von ionisierender Strahlung und bietet aufgrund der rapiden technischen Fortschritte immer mehr und immer genauere Untersuchungsmöglichkeiten an. Auf Basis dieser Entwicklung steigen sowohl die
Untersuchungszahlen als auch die Indikationsstellungen für eine MRT [1]. Parallel dazu vervielfachen sich die Zahlen der implantierbaren Schrittmacher und Defibrillatorsysteme. In den letzten Jahren zeigte sich jedoch, dass SM- und ICD-Patienten mit erheblich weniger MRT-Untersuchungen bei gleicher Indikationsstellung versorgt werden als Nicht-SM/ICD-Träger [2]. Kleine Studien belegen allerdings, dass die Durchführung einer MRT zumeist von klinischem Zusatzwert ist, die Behandlung direkt beeinflusst oder gar ändert [3]. Zusätzlich benötigen ca. 50-75\% der SM/ICD-Träger nach der Implantation im weiteren Leben eine klinisch indizierte MRT [1]. Demzufolge besteht aus den o. g. Gründen die Notwendigkeit, auch SM- und ICD-Patienten den Zugang zu MRT-Untersuchungen und insbesondere deren sichere Durchführung zu ermöglichen.

\section{Potenzielle Interaktionen}

Sowohl das statische Feld als auch die Gradientenfelder und das Radiofrequenz(RF)-Feld beeinflussen unterschiedlich, aber auch teilweise überlappend das implantierte SM- oder ICDSystem des Patienten.

Das statische Feld kann mechanische Zugkräfte bzw. Drehkräfte auf das implantierte System ausüben [4]. Daneben besteht die Gefahr der Interaktion des statischen Felds mit dem SM-System über ein Reset des Systems (Reed-switch-Aktivierung). Des Weiteren besteht das Risiko einer inadäquaten SM-Therapie durch einen im MRT veränderten und falsch interpretierten Rhythmus [5].
$\mathrm{Zu}$ den potenziellen Gefahren, die vom Gradientenfeld ausgehen, zählen Beschädigungen der Elektronik des Systems, ein System-Reset, Vibrationen sowie über die SM-Sonden induzierte Arrhythmien. Zudem können, wie durch das statische Feld, inadäquate Therapien provoziert werden.

Das zugeschaltete Radiofrequenzfeld ist für die gefürchtete Sondenerhitzung verantwortlich. Ferner kann das Ablesen des Rhythmus (Sensing) gestört werden, was wiederum in inadäquaten SM-Aktivierungen resultieren kann.

\section{MRT-Interaktionen in der klinischen Praxis}

Vor Einführung des als absolut essenziell $\mathrm{zu}$ sehenden Monitorings von SM-Patienten während der MRT-Untersuchung wurden in diesem Zusammenhang sogar Todesfälle beschrieben [6]. Aufgrund der technischen Weiterentwicklung sind mittlerweile nur die im Folgenden aufgeführten Probleme mehr oder weniger praxisrelevant.

\section{Verdrehung der SM-Systeme}

In modernen Schrittmachersystemen ist nur in geringem Ausmaß ferromagnetisches Material verbaut. Studien zu 1,5 T zeigten, dass neuere Systeme nur einer geringen Anziehungskraft im statischen Magnetfeld ausgesetzt sind. Aufgrund der unterschiedlichen Bauart weisen jedoch ICD-Systeme höhere Zug- und Drehkräfte auf, sodass bei diesen (speziell bei älteren) Systemen Einflüsse des statischen Felds zu berücksichtigen sind [7]. Inzwischen sind ebenfalls MRc-ICD- 
Systeme am Markt erhältlich, die zumindest 1,5-T-Ganzkörperuntersuchungen erlauben [8].

\section{Erhitzung der SM-Sonden}

Die potenzielle, unerwünschte Funktion der SM-Sonden als Antennen im Radiofrequenzfeld und die daraus resultierende Strominduktion an den Sonden, mit Ableitung in das angrenzende Myokard, kann zur direkten Auslösung von ektopen Herzschlägen bzw. anhaltenden Arrhythmien führen. Zusätzlich könnte eine Erhitzung des an der Sondenspitze angrenzenden Gewebes eine Myokardschädigung bewirken, welche nach ihrer narbigen Abheilung die Überleitung des SM-Impulses an das Myokard behindert (sog. Impedanzerhöhung).

Bei der Messung der absoluten Erhitzung zeigten ältere In-vitro-Studien eine Erwärmung der Sonden auf bis zu $63^{\circ} \mathrm{C}$ (oder $+50^{\circ} \mathrm{C}$ ), wobei nur die Elektrode alleine einer MRT-Sequenz unterzogen wurde. Bei Versuchsanordnung der Elektrode zusammen mit dem Aggregat bzw. Kühlung durch eine Natriumchloridlösung fielen die Unterschiede wesentlich geringer aus und betrugen max. $9^{\circ} \mathrm{C}$ [9]. Insgesamt ist das Abschätzen des Erhitzungspotenzials komplex, da neben dem Sondenmaterial auch Länge und Lage, speziell der gestreckten Sondenanteile in der MRT, als Einflussfaktoren zu sehen sind [10].

In-vivo-Studien, welche Troponin $\mathrm{T}$ als einen Marker des myokardialen Hitzeschadens verwendeten, konnten zum größten Teil keinen relevanten Troponinanstieg nach der MRT mit Schrittmachersystemen nachweisen $[11,12]$. Lediglich in einer Studie von Sommer zeigten 4 von 82 Patienten einen relevanten TroponinT-Anstieg, welcher bei einer Person zu einem Impedanzanstieg an der Sonde im Follow-up nach einigen Monaten führte [13].

\section{Interaktion mit der elektrischen Funktion}

Ältere SM- und ICD-Systeme haben größtenteils einen sog. "Reed switch" verbaut, welcher mittels Ein- und Ausschalten die grundlegenden Funktionen des SM-Geräts steuert. Dieser kann durch einen kleinen Handmagneten aktiviert oder deaktiviert werden. Paradoxerweise ist die Aktivierung oder Deaktivierung mittels eines HochfeldMRT (1,5 oder $3 \mathrm{~T}$ ) nicht voraussehbar, womit wesentliche Funktionen des SM wie asynchrones Pacing, kein Pacing oder Tachyarrythmieerkennung gestört werden können [5].

In der wohl größten Registerstudie (Magnasafe Trial), in der 1500 NichtMRc-SM-Systeme mit 500 ICD-Trägern prospektiv vor und nach einer nichtthorakalen MRT untersucht wurden, konnten lediglich 6(0,6\%) partielle elektrische Resetvorgänge der Systeme beobachtet werden. In einer Metaanalyse ebenfalls mit Nicht-MRc-Geräten, zeigten 1,6\% der untersuchten SM einen Reset, allerdings bei keinem nach 2006 implantierten System [11]. In dieser Analyse konnte lediglich ein einziger signifikanter Abfall der Batteriespannung nachgewiesen werden, welcher allerdings als klinisch nicht relevant einzustufen war [11].

Bisher wurde von lediglich einer inadäquaten Schockabgabe eines akzidentiell untersuchten ICD berichtet. Hier war das Gerät allerdings nicht suffizient auf die MRT-Untersuchung vorbereitet und das ICD-System aktiv [14].

\section{Patientenmonitoring}

Essenziell bei der Durchführung einer MRT ist das adäquate Patientenmonitoring. Dies gilt sowohl für MRc- als auch für Nicht-MRc-Geräte. Das Monitoring muss nach der Lagerung des Patienten auf der MRT-Liege, noch vor der Installation der Sende-/Empfangsspulen eingerichtet werden. Empfohlen sind einerseits die MR-Pulsoxymetrie, andererseits - supplementär dazu - eine EKG-Ableitung. Darüber hinaus ist der Patient zu instruieren, Symptome wie Schwindel, Hitzegefühl oder neu auftretendes Unwohlsein sofort über das Mikrofon an das MRTPersonal zu kommunizieren.

Die MRT-unabhängige, jedoch MRTtaugliche Pulsoxymetrie erlaubt eine gute Überwachung des Patienten und liefert grobe Informationen über den Rhythmus sowie die periphere Sauerstoffsättigung. Ein weiterer Vorteil des Systems ist die Unbeeinflussbarkeit des Signals durch RF-Pulse und Gradientenfeld. Aufgrund der peripheren Ableitung liefert die Pulsoxymetrie jedoch keine genauen Informationen über den Rhythmus des Patienten. Dies ist besonders beim Auftreten von Tachy- oder Bradyarrythmien relevant, welche mit einem peripheren Blutdruck- und Sättigungsabfall einhergehen.

Die EKG-Ableitung alleine wird als insuffizient bewertet, da sie sowohl durch RF-Impulse als auch durch das Gradientenfeld gestört werden kann. Dies ist nicht der Fall bei dezidiert MR-tauglichen externen Überwachungsgeräten, wie z. B. MR-taugliche Anästhesie-Einheiten.

Sollte es zu einem Zwischenfall kommen, ist der sofortige Abbruch der Untersuchung die Bergung des Patienten aus dem MR-Gefahrenbereich einzuleiten. Die Notfallkette muss dem gesamten, an der Untersuchung beteiligten Personal bekannt sein.

Die Verwendung eines Defibrillators in der MR-Gefahrenzone ist aufgrund der Insuffizienz der Geräte und des zu erwartenden Missile-Effekts der NichtMR-tauglichen Defibrillatoren kontraindiziert. Die suffiziente Ladung und die Schockabgabe werden durch das statische Magnetfeld verhindert.

\section{Nicht-MR-taugliche Schrittmacher und ICDs}

Die Durchführung einer MRT-Untersuchung mit nicht dezidiert als MRc ausgewiesenen Geräten stellt aufgrund der inzwischen sehr guten Datenlage keine absolute Kontraindikation mehr dar [15]. Nichtsdestotrotz ist eine MRT in diesem Zusammenhang als "off-label use“ zu sehen und bedarf aus diesem Grund einer strengen Indikationsstellung.

Die Durchführung einer MRT mit Nicht-MRc-Geräten sollte streng einem festgelegten und an das jeweilige Zentrum angepassten Workflow folgen. Die wichtigsten Punkte hierbei sind die Möglichkeit der SM-Überprüfung unmittelbar vor und nach der Untersuchung, die strikte Einhaltung vorgegebener MREinstellungen (Feldstärke, spezifische absorbierte Leistung [SAR], Scanlänge), das Vorhandensein eines adäquaten 
Monitorings des Patienten während der MRT-Untersuchung sowie die Möglichkeit zur raschen medizinischen Intervention durch in Notfallmedizin geschultes Personal. Unter diesen Voraussetzungen können Nicht-MRc-SM/ICD-Systeme als eine relative MRT-Kontraindikation angesehen werden [16].

\section{Indikation}

Da es sich um eine Off-label-Anwendung handelt, muss die Indikation zur MRT interdisziplinär zwischen dem jeweiligen Zuweiser, der Radiologie und der Kardiologie in Konsensus getroffen werden. Der Mehrwert der geplanten Untersuchung muss gegenüber anderen Bildgebungsmodalitäten signifikant sein. Folgende Faktoren müssen zusätzlich in diesen Entscheidungsprozess einfließen und sind mit einem höheren Risiko von Komplikationen behaftet:

- SM-Abhängigkeit des Patienten,

- hohe Narbenlast (vulnerables Myokard), z. B. ischämische Kardiomyopathie, andere strukturelle Herzerkrankungen,

- ICD-Träger mit häufigen Auslösungen der Schockfunktion,

- Vorhandensein von epikardialen Sonden bzw. alten Sondenresten,

- gering eingeschränkter Funktionsstatus des SM-Systems (niedrige Batterie und höherer Sondenthreshold),

- Untersuchungsregion und Spulenpositionierung (thorakal vs. extrathorakal),

- Implantationszeitpunkt des SM (vor 1998) bzw. des ICD-Systems (vor 2000; [17]).

Das höchste Risiko geht hierbei von älteren SM- und ICD-Systemen in Kombination mit einem vulnerablen Patienten mit hoher Narbenlast und/oder ausgedehnter struktureller Herzerkrankung aus. In diesem Zusammenhang werden MRT-Untersuchungen, bei Zutreffen des o. g. Implantationsdatums sowie eines fehlenden asynchronen Modus, nach Empfehlungen aus dem angloamerikanischen Raum nach wie vor als absolut kontraindiziert angesehen [17].

Radiologe 2019 · 59:885-893 https://doi.org/10.1007/s00117-019-0567-9

(c) Der/die Autor(en) 2019

\section{Beitzke}

\section{Sichere MRT-Untersuchung bei Patienten mit Herzschrittmacher und ICD}

Zusammenfassung

Hintergrund. Die Magnetresonanztomographie (MRT) bei Patienten mit Herzschrittmachern und Defibrillatorsystemen bedarf besonderer Voraussetzungen, um die Patientensicherheit während der Untersuchung zu gewährleisten. Fragestellung. Können Patienten mit Schrittmacher und implantierbarem Kardioverter-Defibrillator (ICD) in der MRT sicher untersucht werden?

Material und Methoden. Der Beitrag gibt eine Übersicht über Interaktionen zwischen Schrittmacher und MRT-Systemen, fasst die aktuelle Studienlage zusammen und beschreibt, wie man eine sichere MRT-Unter- suchung bei Patienten mit Schrittmacher und ICD durchführt.

Schlussfolgerung. Die MRT-Untersuchung bei Patienten mit Schrittmacher und ICD kann unter ganz bestimmten Voraussetzungen und unter Einhaltung eines dezidierten Ablaufs sicher durchgeführt werden und sollte daher dem Patienten bei entsprechender Indikation angeboten werden.

Schlüsselwörter

Herzerkrankungen · Implantierbarer Kardioverter-Defibrillator - Magnetresonanztomographie - Interaktionen . Monitoring

\section{Safe MRI examinations in patients with pacemakers and ICD}

\section{Abstract}

Background. Examining patients with cardiac pacemakers and implantable cardioverter-defibrillator systems (ICD) requires special cautions und preparations in order to assure patient safety.

Objectives. Is it possible for pacemaker and ICD patients to safely undergo MRI examination?

Materials and methods. This review provides information on interaction between the pacemaker and MRI systems, provides a review on the evidence published so far and describes how to perform a safe MRI examination in a patient with an implanted pacemaker device.

Conclusion. MRI in patients with cardiac pacemaker and ICD systems is safe under specified conditions and should be offered to patients with a given indication for a MRI.

Keywords

Heart diseases - Implantable cardioverterdefibrillators - Magnetic resonance imaging . Interactions · Monitoring

\section{Aufklärung des Patienten}

Der Patient muss mit adäquatem Abstand zur Untersuchung über die möglichen Risiken der MRT-Untersuchung im Offlabel-Setting informiert werden und sein schriftliches Einverständnis dazu geben. Erörtert werden müssen sämtliche seltene, aber mögliche Komplikationen, von SM/ICD-Fehlfunktionen über Batterieentleerungen, bis hin zu möglichen Gewebeverletzungen durch Erhitzung bzw. der Indiktion von Arrhythmien durch den MRT-Scan. Zusätzlich muss der Patient darüber informiert werden, dass das SM-System im MRT nur eingeschränkt funktioniert und daher die Reaktion auf potenziell auftretende Arrhythmien inadäquat sein kann.

\section{Modus des Schrittmachers im MRT}

Der Modus des SM-Systems wird je nach Eigenrhythmus oder SM-Abhängigkeit des Patienten gewählt. Am häufigsten wird das asynchrone Pacing auch bei nicht-SM-abhängigen Patienten empfohlen. Bei SM-abhängigen Patienten wird ein permanentes Pacing mit einer voreingestellten Herzrate empfohlen [15]. Schockfunktion und andere SMZusatzfunktionen werden deaktiviert. 
Durchführung der MRT mit Nicht-MRc-Geräten

Im Gegensatz zu den nachfolgend beschriebenen MRc-Geräten ist bei den nicht dezidierten MRc-Geräten erhöhte Vorsicht geboten. Folgende Maßnahmen sind hier vor und nach der MRT relevant [16]:

- schriftliche Dokumentation des Ablaufs inklusive der SM-Parameter vor und nach der Untersuchung,

- Anwesenheit eines Kardiologen während der Untersuchung,

- Monitoring mittels Pulsoxymetrie und EKG,

- Notfallausrüstung vor Ort (Defibrillator, EKG und Kreislaufmonitoring außerhalb der MRT),

- Programmiereinheit des SM oder ICD greifbar,

- in Notfallsituationen geschultes Personal in Rufbereitschaft,

- Anforderung an die MRT-Untersuchung:

- geschlossenes 1,5 T System, aktive MR-Zeit $<30$ min,

- Ganzkörper-SAR $<2 \mathrm{~W} / \mathrm{kg}$ oder Kopf SAR $<3,2 \mathrm{~W} / \mathrm{kg}$.

Sowohl bei Nicht-MRc-Geräten als auch bei MRc-Geräten, empfiehlt sich dringend die schriftliche Dokumentation der einzelnen Schritte vor, während und nach der Untersuchung. Vorlagen hierfür werden entweder von den SMHerstellern oder Fachgesellschaften bereitgestellt [18]. Diese sollten allerdings an den lokalen Ablauf des jeweiligen Zentrums angepasst werden.

\section{Monitoring des Patienten}

Das unabdingbare Monitoring des Patienten unterscheidet sich nicht von dem Monitoring mit MRc-Geräten, ist aber aufgrund des „off-label use“ noch genauer einzuhalten (s. oben). Die Anwesenheit eines Kardiologen während der Untersuchung ist zu empfehlen.

\section{Anforderung an die} MRT-Untersuchung

Das Gros der Studien mit Nicht-MRcGeräten wurde an geschlossenen 1,5-T-
Systemen durchgeführt, weshalb hier die größte Evidenz existiert und diese Feldstärke, sofern möglich, zu bevorzugen ist [19]. Lokale Sendespulen, insbesondere im Thoraxbereich, sollten vermieden werden. Als Sendespule sollte die im Magneten integrierte Bodyspule dienen. Lokale Empfangsspulen (Kopf, Knie) stellen kein Problem dar.

Um die, wie oben erwähnt, schwer abzuschätzende Gefahr der Erhitzung der SM/ICD-Sonden zu minimieren, ist ein SAR-Limit von $2 \mathrm{~W} / \mathrm{kg}$ beim Ganzkörper bzw. 3,2 W/kg bei Kopfuntersuchungen einzuhalten. Zudem sollte die absolute Scanzeit von 30 min nicht überschritten werden. Diese Maßnahme soll im Fall einer dennoch stattfindenden Erhitzung der Sonden größeren Schaden am Myokard durch prolongierte Einwirkung der Hitze verhindern.

\section{Reprogrammierung des Schrittmachers}

Die Reprogrammierung und eine Systemüberprüfung des Nicht-MRc-SM/ICDSystems sollte unmittelbar nach der MRT-Untersuchung, idealerweise nahe dem MRT-Gerät erfolgen. Eine weitere Untersuchung, insbesondere der Sondenimpedanz, sollte nach etwa 3 Monaten vorgenommen werden [13].

\section{MRT-taugliche Geräte}

Alle größeren SM-Hersteller haben inzwischen MRc-Geräte in ihrem Produktportfolio. Diese Geräte erlauben in Kombination mit den jeweiligen Sonden desselben Anbieters sichere MRT-Untersuchungen unter bestimmten Voraussetzungen. $\mathrm{Zu}$ beachten sind die herstellerabhängigen Unterschiede in der Freigabe des SM betreffend Feldstärke und Untersuchungsregion, wobei der Thorax als Scanregion sowie höhere Feldstärken meist nicht erlaubt sind. Neue Geräte bieten immer häufiger auch die Möglichkeit von 3-T-Untersuchungen und/oder ohne Restriktionen der Scanregion an. Dies gilt inzwischen teilweise auch für moderne ICD-Systeme [20]. Auch die vom SM-Hersteller freigegebenen SAR-Level sind für diese Geräte teilweise auf bis zu
$4 \mathrm{~W} / \mathrm{kg}$ angestiegen, was der Betriebsart 1. Ordnung entspricht.

\section{Aufklärung des Patienten/ Ablaufdokumentation}

Obwohl es sich um einen „in-label use“ handelt, sollte der Patient über Interaktionen zwischen dem MRT-Umfeld und dem implantierten SM-System aufgeklärt werden. Dabei soll allerdings die durch Studien unterlegte Sicherheit dieser Untersuchung betont werden [20-22]. Hierbei ist jedoch zu beachten, dass lediglich die Kombination aus MRc-SM-System und MRc-Sonden einen „in-label use“ erlauben. Patienten, welche z. B. ein neues Aggregat auf ältere Sonden erhalten haben, gehören somit per definitionem nicht mehr zu dieser Gruppe. Ähnlich wie bei Nicht-MRc-Geräten empfiehlt sich die schriftliche Dokumentation der Untersuchung (Freigabe Kardiologie, Untersuchung durch Radiologie und Reprogrammierung Kardiologie; [18]).

\section{Vor der Untersuchung}

Alte, aufgelassenen Sonden bzw. retinierte epikardiale SM-Sonden von temporären SM-Systemen stellen ebenfalls laut sämtlichen Herstellern ein Hindernis für den „in-label use“ der MRc-Geräte dar, da sie als zusätzliche Antenne im RFFeld fungieren können. Diese sollten mittels eines Thorax-Röntgens ausgeschlossen werden (vgl. Abschn. „Spezielle Untersuchungssituationen").

Bei neu implantierten Systemen wird im Allgemeinen eine Wartezeit von bis zu 6 Wochen bis zur Durchführung einer MRT empfohlen, um ein sicheres Einheilen der Sonden zu gewährleisten.

\section{Umstellung des SM-Systems in den MR-Modus}

Die Umstellung des SM-Systems in den MR-Modus muss in einem vom Hersteller vordefinierten Zeitraum erfolgen. Geräte der neueren Generation sind teilweise schon in der Lage, das Magnetfeld selbstständig zu detektieren, womit ein zuvor programmierter MR-Modus automatisch aktiv wird. Beim Verlassen des MR-Felds stellt sich das Gerät auf den 
Tab. 1 Kontaktseiten der Hersteller zum Geräteabgleich vor der MRT-Untersuchung.

(Stand: Ende April 2019)

Medtronic Surescan

http://wwwp.medtronic.com/mrc/

Biotronik

Abbott (früher St. Jude Medical)

Miroport (früher Sorin und/oder LivaNova)

normalen Modus zurück. Dies hat den Vorteil, dass sämtliche SM-Funktionen bis zum definitiven Betreten des Magnetfelds dem Patienten bei Bedarf zur Verfügung stehen. Zudem können z. B. bei telemedizinisch betreuten Patienten die Nachfolgeuntersuchungen entfallen, da das SM-System in der Lage ist, eine Systemüberprüfung an den betreuenden Kardiologen zu schicken. Bei Systemen, die keine automatische Detektion des Magnetfelds beinhalten, muss eine Umstellung in den MR-Modus in der Regel unmittelbar vor der Untersuchung erfolgen.

Um einen für den Patienten angenehmen und effizienten Ablauf der MRT-Untersuchung zu gewährleisten, sollten die Details des SM/ICD-Systems schon bei der Terminvereinbarung abgeklärt werden. Dies ist bei der Wahl der Feldstärke hilfreich und erlaubt zudem, das Scanprotokoll, falls notwendig, vorher auf vorliegende SM-Restriktionen anzupassen. Am Tag der Untersuchung sollte im Idealfall nur die Um- bzw. Rückstellung des SM-Geräts erfolgen. Ein derart optimierter Ablauf gestattet es, den Patientendurchlauf zu erhöhen und gleichzeitig die Wartezeiten zu reduzieren [23].

\section{Durchführung der MRT mit MRc-Geräten}

Beim Monitoring ist ident zu Nicht-MRcGeräten vorzugehen. MR-unabhängige Pulsoxymetrie bzw. supplementär dazu ein EKG (s. oben) sind obligatorisch. Die Anwesenheit eines Kardiologen ist nicht zwingend erforderlich, das Personal sollte jedoch mit der Notfallkette vertraut sein [24].

Die MRT-Parameter bzw. die Freigaben der Untersuchungsgebiete von den jeweiligen Herstellern variieren. Relevante Parameter inkludieren wiederum: Exklusionszone), MRT-Systems, (Slew Rate, Gradientenstärke), use" gelten.

\section{Herstellerkontaktseiten}

Die in $\bullet$ Tab. 1 aufgeführte Liste beinhaltet die Kontaktseiten der Hersteller zum Geräteabgleich vor der MRT-Untersuchung (Stand: Ende April 2019).

\section{Spezielle Untersuchungs- situationen}

Ganzkörper- vs. Nicht-Ganzkörper-

- Feldstärke und Bauart des geeigneten

- Eigenschaften des Gradientensystems

- keine anderen Implantate, die als Kontraindikation für einen ,in-label

Die meisten MRc-Geräte erlauben die Durchführung der MRT-Untersuchungen jeweils mit einer SAR-Grenze von $2 \mathrm{~W} / \mathrm{kg}$ bei Körperuntersuchungen und von $3,2 \mathrm{~W} / \mathrm{kg}$ bei Kopfuntersuchungen jeweils mit normalem Betriebsmodus. Einzelne Systeme lassen darüber hinaus Untersuchungen, sowie mit einer SARObergrenze von inzwischen $4 \mathrm{~W} / \mathrm{kg} \mathrm{zu}$. Die meisten Systeme akzeptieren ausschließlich die Verwendung von Empfangsspulen, lediglich einzelne Hersteller erlauben den Einsatz von kombinierten Sende- und Empfangsspulen, zumeist in der Peripherie (z.B. Knie, untere Extremitäten). Andere von den Herstellern vorgegebene Einschränkungen betreffen oft die Scanzeit pro Sequenz, die minimale Körpergröße sowie das Vorhandensein einer erhöhten Körpertemperatur.

Nach der Durchführung sollte eine schriftliche Dokumentation des gesamten Ablaufs und der Reprogrammierung des SM/ICD erfolgen, Letzteres nur, falls keine automatische MR-Feld-Detektion im Gerät eingebaut ist. Die notwendige Reprogrammierung in Kombination mit einer Systemüberprüfung sollte idealerweise direkt nach der Untersuchung, (aber nicht zwingend im MRT-Vorbereitungsraum) spätestens jedoch am selben Tag stattfinden. Eine Systemüberprüfung nach 3 Monaten wie für Nicht-MRc-Geräte ist nicht zwingend erforderlich.

\section{Patienten mit einem ICD-System}

Patienten mit einem ICD unterscheiden sich von normalen rhythmologischen SM-Patienten zumeist durch das Vorhandensein einer strukturellen Herzerkrankung mit oder ohne signifikanter Narbenbildung. Aufgrund der Tatsache, dass eine Ladung und Schockabgabe des Defibrillators im statischen MRFeld nicht suffizient möglich ist, sind die Schockfunktionen des internen Defibrillators während der Untersuchung deaktiviert. Daher muss dem betreuenden Personal bewusst sein, dass hier eine vulnerable Patientengruppe vorliegt. Die kritische Evaluierung der MRT-Indikation ist bei dieser Patientengruppe daher essenziell.

\section{Nicht-MRc-ICD}

ICD-Systeme, welche vor dem Jahr 2000 implantiert wurden, stellen eine absolute Kontraindikation dar [17]. Bei Systemen nach diesem Baujahr ist der Patient insbesonders über die Möglichkeit einer potenziellen permanenten Malfunktion des SM Systems zu informieren. Darüber hinaus muss über Risiken wie Sondenfehlfunktion, Erhitzung (bei Vorliegen von drei Sonden) und daraus resultierende eventuelle Erhöhungen der Impedanz an der Sondenspitze aufgeklärt werden.

Es ist $\mathrm{zu}$ beachten, dass im Thoraxbereich die Bildqualität durch die Schockspulen im Bereich der Vena cava superior bzw. das meist links gelegene Aggregat deutlich beeinträchtigt werden kann. Dies gilt vor allen für Herz-MRTUntersuchungen.

\section{MRc-ICD}

Da es sich hierbei um einen ,in-label use“ handelt, ist die Gefahr der ICD-Fehlfunk- 

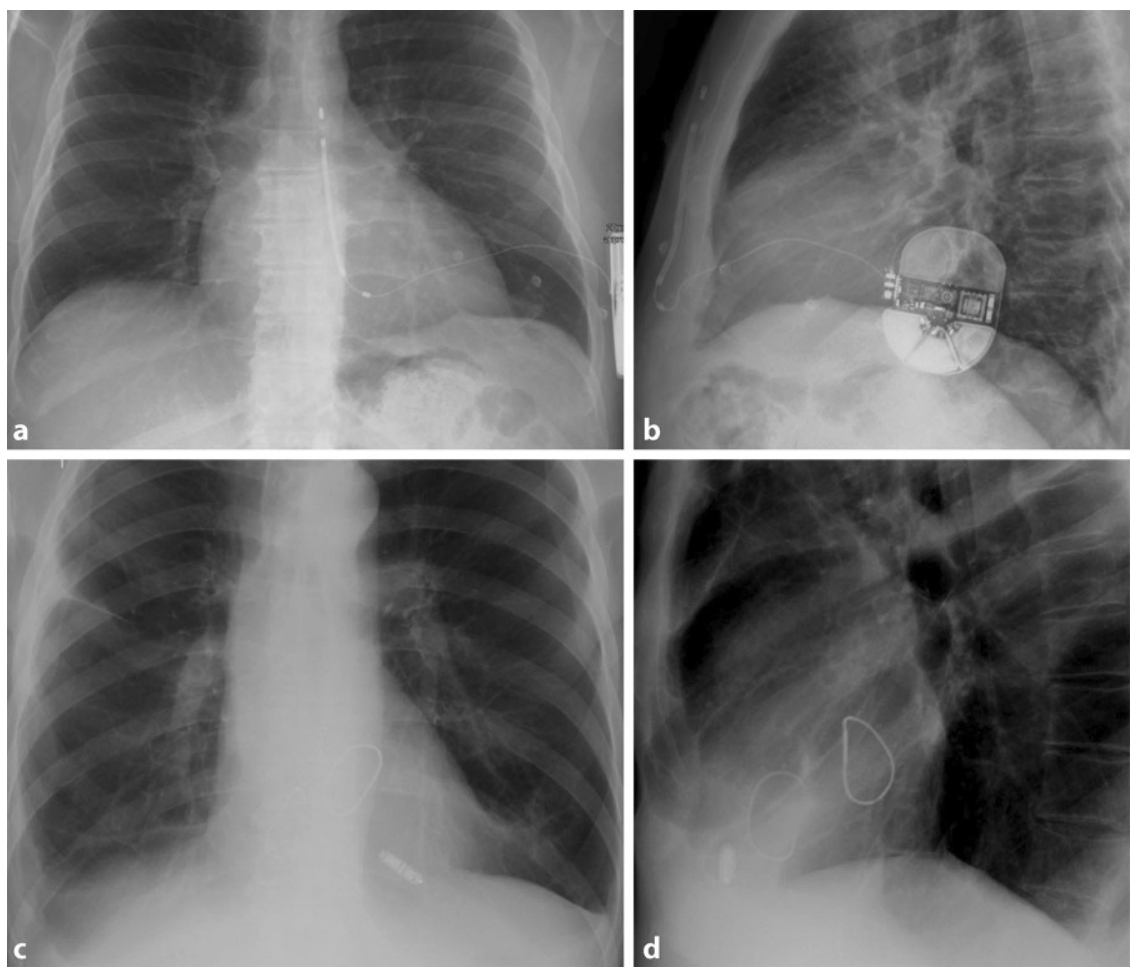

Abb. $1 \Delta$ a, b Subkutanes Implantierbarer-Kardioverter-Defibrillatoren-System mit der prästernal gelegenen Schocksonde und dem Generator links thorakolateral. c, d Sondenloses Schrittmachersystem mit dem Aggregat in der rechtsventrikulären Spitze

tion nach einer MRT-Untersuchung als sehr gering einzuschätzen. Dem Zuweiser, sowie dem durchführenden Personal, müssen jedoch die Risiken der teilweise fehlenden ICD-Funktionen bekannt sein. Hier ist im Vorfeld wiederum die Nutzen-Risiko-Abwägung kritischer zu führen als bei konventionellen SM-Trägern. Ein Patient, dessen ICD wöchentlich auslöst, ist wahrscheinlich heikler zu sehen als ein Patient, dessen ICD noch nie in Funktion treten musste.

\section{Subkutane Defibrillatoren}

Bei dieser Bauart eines Defibrillators (Boston Scientific) ist die Schocksonde im Bereich des Sternums subkutan und wird nicht transvenös eingebracht. Dieses System ist für 1,5-T-Untersuchungen freigegeben ([8]; - Abb. 1a, b).

\section{Sondenlose Schrittmacher}

Die sondenlosen Herzschrittmacher, sog. "leadless pacemaker" (Medtronic Mi(ra), werden transvenös eingebracht und mit Hilfe von Ankersystemen in den api- kalen Trabekeln des rechten Ventrikels befestigt (• Abb. 1c, d). Dieses System ist ausschließlich für die Ventrikelstimulation gebaut und kommt daher ohne Vorhofsonde aus. Für die MRT-Untersuchung ist das System MR-tauglich bis $3 \mathrm{~T}$ unter bestimmten Scanbedingungen und zuvor erfolgter Umstellung in den MR-Modus (• Abb. 1a, b).

\section{MRT mit aufgelassenen SM-Sonden bzw. epikardialen Sondenresten}

Nach einem Wechsel des SM-Aggregats aufgrund oder nach erfolgter Herztransplantation verbleiben unter Umständen aufgelassene Sonden, Sondenreste, Defibrillatorreste bzw. auch epikardiale SMSondenreste in den Patienten. Diesem Patientenkollektiv wird der Zugang zum MRT damit erheblich erschwert, da die gewiesen werden [6].

Im Fall eines neu implantierten, MRc-SM stellen Implantatreste eine Kontraindikation für einen ,in-label use" dar. Auch bei Nicht-MRc-Geräten Patienten seitens der Radiologie oft ab- wird aufgrund der zusätzlich vorhandenen Antenne von einer Untersuchung eher abgeraten [16, 24]. In-vitro-Experimente zeigen, dass teilweise erhebliches Erhitzungspotenzial (bis +50 Grad) vorhanden ist, welches zusätzlich aufgrund der immer unterschiedlichen Konfiguration der Sondenreste im Patienten sehr schwer vorauszusagen ist [25]. Demgegenüber stehen einzelne, teilweise auch retrospektive Studien, welche über kein erhöhtes Risiko bei MRT-Untersuchungen berichten. Dies betrifft sowohl SMSondenreste nach Transplantation als auch SM-Träger mit aufgelassenen Sonden $[26,27]$. Wichtig hierbei erscheint jedoch zusätzlich die Optimierung des MRT-Protokolls mit Fokus auf Scanzeit und SAR sowie die ausführliche Aufklärung des Patienten [28].

\section{3-T-Untersuchungen}

Die Sicherheit von 1,5-T-Untersuchungen bei Patienten mit SM- und ICDGeräten ist in der Literatur breit belegt. Bei 3-T-Untersuchungen ist die Datenlage deutlich geringer $[29,30]$. Neuere SM, aber auch ICD-Systeme, werden zunehmend auch für $3 \mathrm{~T}$ freigegeben sein, wenn auch initial gelegentlich mit Restriktionen der Scanregionen am oder nahe des Thorax.

\section{Herz-MRT mit SM/ICD-Systemen}

Bei der Herzbildgebung rückt neben der Sicherheit des Patienten auch der Aspekt der diagnostischen Bildqualität in die Entscheidungsfindung, ob der Patient mittels MRT untersucht werden soll oder nicht. Diese muss gewährleistet sein, um die mit der Untersuchung verbundenen Risiken zu rechtfertigen. Die Bildqualität wird maßgeblich von Patientenfaktoren wie Größe und Breite des Thorax und der daraus resultierenden Lage des SM/ICD-Generators im Verhältnis zum Herzen beeinflusst. ICDSysteme sind links pektoral positioniert und zusätzlich mit Schocksonden im Bereich der Vena cava superior und des rechten Ventrikels ausgestattet, welche sowohl die Funktionsaufnahmen als auch die Late-Gadolinium-Enhance- 


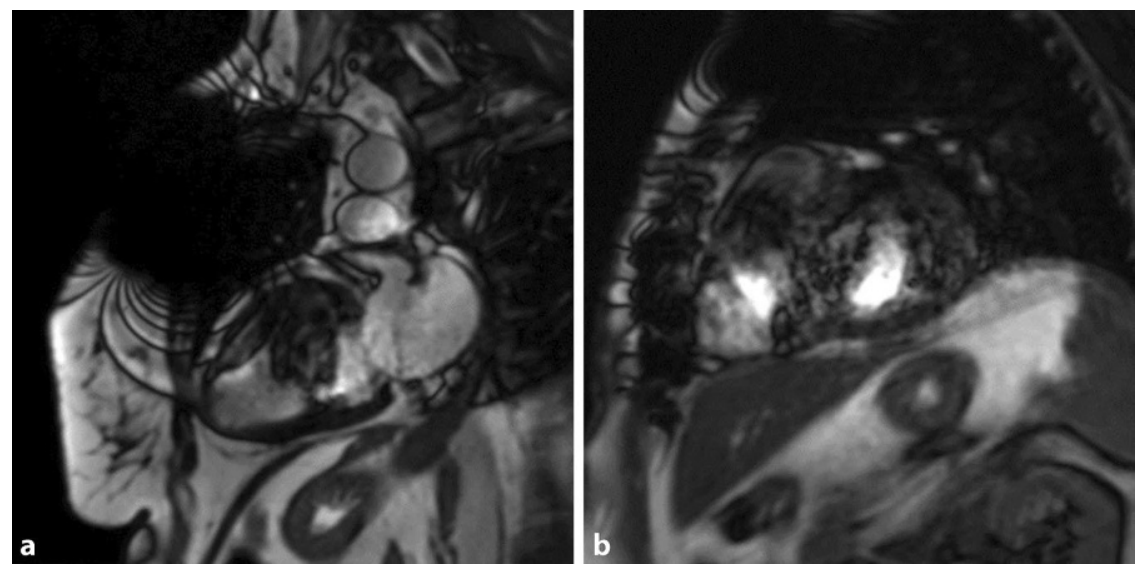

Abb. 2 ム Deutlich reduzierte, nichtdiagnostische Bildqualität der Herz-MRT (a, b bSSFP-Sequenzen [,"balanced steady-state free precession“]) bei einem Patienten mit linksseitigem Implantierbarer-Kardioverter-Defibrillatoren-Generator
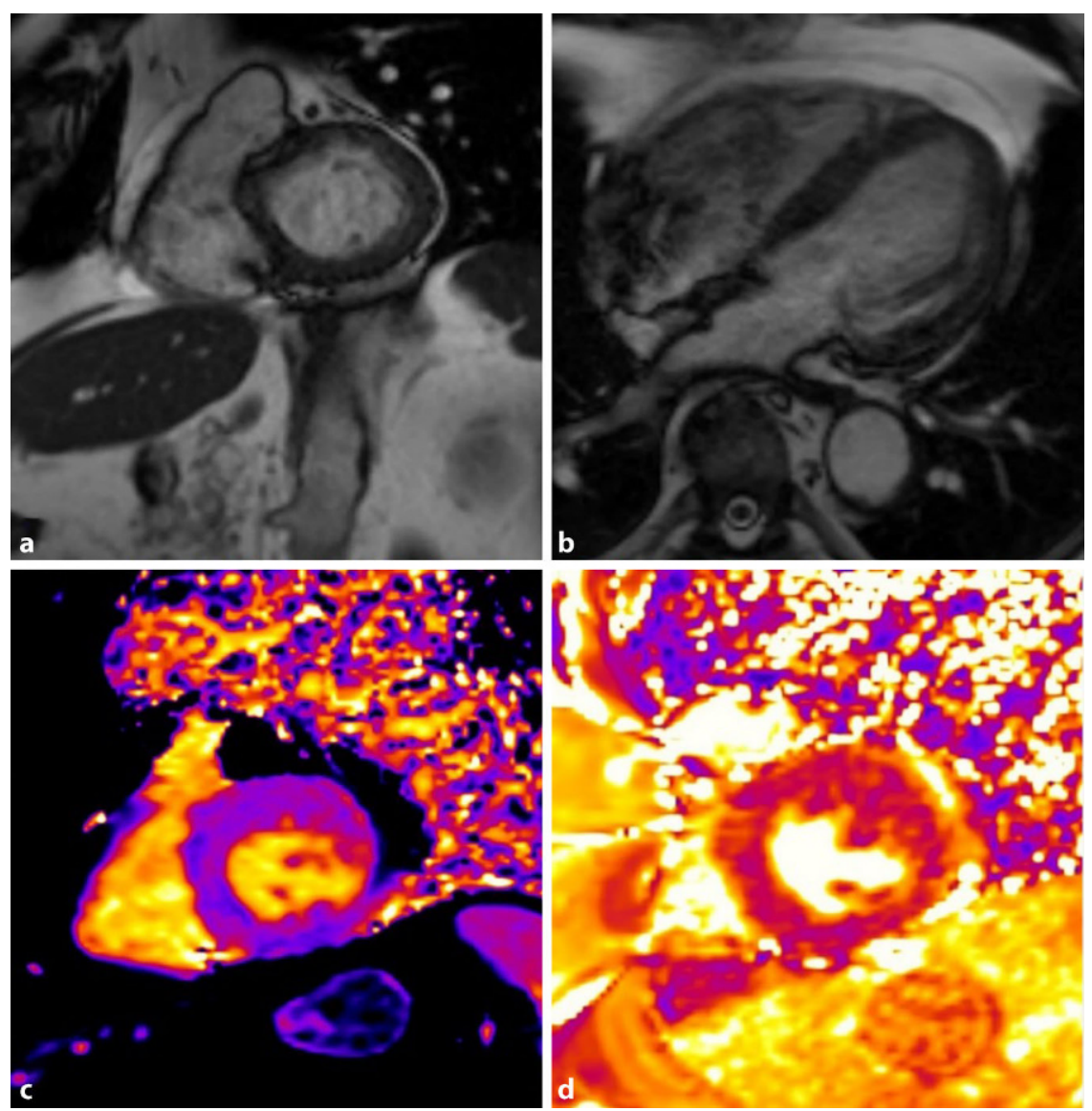

Abb. 3 A Erhaltene Herz-MRT-Bildqualität bei Schrittmacher und rechtsseitigem Generator. a, b Geringe Artefaktbildung derbSSFP-Sequenzen („,balanced steady-state free precession“).c, $\mathbf{d}$ keine sichtbaren Artefakte auf den Mapping-Sequenzen
ment-Bilder stark negativ beeinflussen (- Abb. 2 und 3).

Der Einsatz von neuen Breitbandsequenzen kann diese Artefakte jedoch suffizient unterdrücken, sodass auch bei ungünstiger Anatomie diagnostische Bildqualität erreicht werden kann [31]. Dies ist insbesondere bei der Narbenbildgebung vor rhythmologischer Intervention von hoher klinischer Relevanz [32].

\section{Diskussion}

Patienten mit implantierten SM/ICDGeräten sind tendenziell immer noch von der MRT-Bildgebung ausgeschlossen und damit zum Teil diagnostisch nicht optimal versorgt, obwohl der Bedarf und auch die Wertigkeit der MRT bei diesen Patienten gut belegt sind [1, 3].

Die Datenlage zeigt, dass die sichere MRT-Untersuchung mit implantierbaren Schrittmachen und teilweise auch ICD-Geräten sowohl mit Nicht-MRc-als auch mit MRc-Geräten unter Beachtung gewisser Voraussetzungen möglich ist. Dazu gehören ein dezidierter, den lokalen Gegebenheiten angepasster Workflow, von Kardiologen festgelegte SM/ICDEinstellungen, an den jeweiligen SM angepasste Scanprotokolle, das Monitoring des Patienten sowie enge interdisziplinäre Zusammenarbeit. Unter Einhaltung dieser Sicherheitsrestriktionen ist die Untersuchung mit einem sehr geringen Risiko behaftet und sollte Patienten daher bei valider Indikationsstellung nicht vorenthalten werden.

\section{Fazit für die Praxis}

- Ein Großteil der MRT-Untersuchungen kann den Patienten trotz implantierten Schrittmachersystemen angeboten werden.

- Die MRT muss mit definierten Schrittmachereinstellungen und unter Monitoring des Patienten erfolgen.

- Bedingt MRT-taugliche („MR conditional') Systeme erlauben In-labeluse-Untersuchungen mit sehr geringem Risiko. 


\section{Korrespondenzadresse}

\section{Dr. D. Beitzke}

Universitätsklinik für Radiologie und

Nuklearmedizin, Medizinische Universität Wien Währinger Gürtel 18-20, 1090 Wien, Österreich dietrich.beitzke@meduniwien.ac.at

Funding. Open access funding provided by Medical University of Vienna.

\section{Einhaltung ethischer Richtlinien}

Interessenkonflikt. D. Beitzke gibt an, dass kein Interessenkonflikt besteht.

Für diesen Beitrag wurden von den Autoren keine Studien an Menschen oder Tieren durchgeführt. Für die aufgeführten Studien gelten die jeweils dort angegebenen ethischen Richtlinien.

Open Access Dieser Artikel wird unter der Creative Commons Namensnennung 4.0 International Lizenz (http://creativecommons.org/licenses/by/4.0/deed. de) veröffentlicht, welche die Nutzung, Vervielfältigung, Bearbeitung, Verbreitung und Wiedergabe in jeglichem Medium und Format erlaubt, sofern Sie den/die ursprünglichen Autor(en) und die Quelle ordnungsgemäß nennen, einen Link zur Creative Commons Lizenz beifügen und angeben, ob Änderungen vorgenommen wurden.

\section{Literatur}

1. Kalin R, Stanton MS (2005) Current clinical issues for MRI scanning of pacemaker and defibrillator patients. Pacing Clin Electrophysiol 28:326-328

2. Nazarian S, Reynolds MR, Ryan MP et al (2016) Utilization and likelihood of radiologic diagnostic imaging in patients with implantable cardiac defibrillators. J Magn Reson Imaging 43:115-127

3. Samar H, Yamrozik JA, Williams RB et al (2017) Diagnostic value of MRI in patients with implanted pacemakers and implantable cardioverterdefibrillators across a cross population: Does the benefit justify the risk? A proof of concept study. JACC Clin Electrophysiol 3:991-1002

4. Schick F (2019) MRT - Wechselwirkungen mit magnetisch aktivem und elektrisch leitfähigem Material. Radiologe. https://doi.org/10.1007/ s00117-019-0514-9

5. Luechinger R, Duru F, Zeijlemaker VA et al (2002) Pacemaker reed switch behavior in $0.5,1.5$, and 3.0 tesla magnetic resonance imaging units: Are reed switches always closed in strong magnetic fields? Pacing Clin Electrophysiol 25:1419-1423

6. Ferris NJ, Kavnoudias H, Thiel C, Stuckey S (2007) The 2005 Australian MRI safety survey. AJR Am J Roentgenol 188:1388-1394

7. Luechinger R, Duru F, Scheidegger MB et al (2001) Force and torque effects of a 1.5-Tesla MRI scanner on cardiac pacemakers and ICDs. Pacing Clin Electrophysiol 24:199-205

8. Migliore F, Mattesi G, De Franceschi $P$ et al (2019) Multicentre experience with the secondgeneration subcutaneous implantable cardioverter defibrillator and the intermuscular two- incision implantation technique. J Cardiovasc Electrophysiol.https://doi.org/10.1111/jce.13894

9. Achenbach S, Moshage W, Diem B et al (1997) Effects of magnetic resonance imaging on cardiac pacemakers and electrodes. Am Heart J 134:467-473

10. Mattei E, Triventi M, Calcagnini G et al (2008) Complexity of MRI induced heating on metallic leads: Experimental measurements of 374 configurations. Biomed Eng Online 7:11

11. Shah AD, Morris MA, Hirsh DS et al (2018) Magnetic resonance imaging safety in nonconditional pacemaker and defibrillator recipients: A metaanalysis and systematic review. Heart Rhythm 15:1001-1008

12. Mollerus M, Albin G, Lipinski M, Lucca J (2008) Cardiac biomarkers in patients with permanent pacemakers and implantable cardioverter-defibrillators undergoing an MRI scan. Pacing Clin Electrophysiol 31:1241-1245

13. Sommer T, Naehle CP, Yang A et al (2006) Strategy for safe performance of Extrathoracic magnetic resonance imaging at 1.5 tesla in the presence of cardiacpacemakersinnon-pacemaker-dependent patients. Circulation 114:1285-1292

14. Atarl,BalU, Ertan Ç etal (2016) Inappropriate shock and battery switching to "end of life" in a patient with biventricular ICD during magnetic resonance imaging. Turk Kardiyol Dern Ars 44:79-81

15. Brignole $M$, Auricchio A, Baron-Esquivias $G$ et al (2013) 2013 ESC Guidelines on cardiac pacing and cardiac resynchronization therapy: The task force on cardiac pacing and resynchronization therapy of the European Society of Cardiology (ESC). Developed in collaboration with the European Heart Rhythm Association. Eur Heart J 34:2281-2329

16. Sommer $T$, Luechinger R, Barkhausen $\mathrm{J}$ et al (2015) German Roentgen Society statement on MR imaging of patients with cardiac pacemakers. Rofo 187:777-787

17. Miller JD, Nazarian S, Halperin HR (2016) Implantable electronic cardiac devices and compatibility with magnetic resonance imaging. J Am Coll Cardiol 68:1590-1598

18. Nuernberg M (2017) Empfehlungen der ÖKG: MRI und SM: Praktisches Vorgehen bei MRUntersuchungen von Schrittmacherpatienten. JKardiol Austrian J Cardiol 24:106-108

19. Nordbeck P, Ertl G, Ritter O (2015) Magnetic resonance imaging safety in pacemaker and implantable cardioverter defibrillator patients: How far have we come? Eur Heart J 36:1505-1511

20. Gold MR, Sommer T, Schwitter J et al (2015) Full-body MRI in patients with an implantable cardioverter-defibrillator: Primary results of a randomized study. J Am Coll Cardiol 65:2581-2588

21. Wollmann CG, Thudt K, Kaiser B et al (2014) Safe performance of magnetic resonance of the heart in patients with magnetic resonance conditional pacemaker systems: The safety issue of the ESTIMATE study. J Cardiovasc Magn Reson 16:30

22. Rosenthal L, McCotter C, Bailey WM et al (2015) Clinical safety of the ProMRI pacemaker system in patients subjected to thoracic spine and cardiac 1.5-T magnetic resonance imaging scanning conditions. Heart Rhythm 13:464-471

23. Bhuva AN, Feuchter P, Hawkins A et al (2019) MR for patients with cardiac implantable electronic devices: Simplifying complexity with a "one-stop" service model. BMJ Qual Saf. https://doi.org/10. 1136/bmjqs-2018-009079

24. Sommer T, Bauer W, Fischbach K et al (2017) MR imaging in patients with cardiac pacemakers and implantable cardioverter defibrillators. Rofo 189:204-217

25. Bonnemains L, Barbier T, Felblinger J (2016) Metal wires should not be abandoned inside implantable cardioverter-defibrillators leads during heart transplantation! Transpl Int 29:1136-1138

26. Austin CO, Landolfo K, Parikh PP et al (2017) Retained cardiac implantable electronic device fragments are not associated with magnetic resonance imaging safety issues, morbidity, or mortality after orthotopic heart transplant. Am Heart J 190:46-53

27. Padmanabhan D, Kella DK, Mehta R et al (2018) Safety of magnetic resonance imaging in patients with legacy pacemakers and defibrillators and abandoned leads. Heart Rhythm 15:228-233

28. Horwood L, Attili A, Luba F et al (2017) Magnetic resonance imaging in patients with cardiac implanted electronic devices: Focus on contraindications to magnetic resonance imaging protocols. Europace 19:812-817

29. Naehle CP, Meyer C, Thomas D et al (2008) Safety of brain 3-T MR imaging with transmit-receive head coil in patients with cardiac pacemakers: Pilot prospective study with 51 examinations. Radiology 249:991-1001

30. Gimbel JR (2008) Magnetic resonance imaging of implantable cardiac rhythm devices at 3.0 tesla. Pacing Clin Electrophysiol 31:795-801

31. Rashid S, Finn JP, Shivkumar Ketal (2014) Improved late gadolinium enhancement MR imaging for patients with implanted cardiac devices. Radiology 270:269-274

32. Singh A, Kawaji K, Goyal N et al (2019) Feasibility of cardiac magnetic resonance wideband protocol in patients with implantable cardioverter defibrillators and its utility for defining scar. Am J Cardiol 123:1329-1335 
Hier steht eine Anzeige.

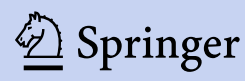

